\title{
Nadège Veldwachter, Littérature francophone et mondialisation
}

Jean-François Plamondon

\section{(2) OpenEdition}

10 Journals

\section{Édition électronique}

URL : https://journals.openedition.org/studifrancesi/3235

DOI : 10.4000/studifrancesi.3235

ISSN : 2421-5856

\section{Éditeur}

Rosenberg \& Sellier

\section{Édition imprimée}

Date de publication : 1 juillet 2013

Pagination : 494-495

ISSN : 0039-2944

\section{Référence électronique}

Jean-François Plamondon, « Nadège Veldwachter, Littérature francophone et mondialisation », Studi

Francesi [En ligne], 170 (LVII | II) | 2013, mis en ligne le 30 novembre 2015, consulté le 02 février 2023. URL : http://journals.openedition.org/studifrancesi/3235; DOI : https://doi.org/10.4000/studifrancesi. 3235

Ce document a été généré automatiquement le 2 février 2023.

\section{(c) (ㅇ) $\odot$}

Creative Commons - Attribution - Pas d'Utilisation Commerciale - Pas de Modification 4.0 International - CC BY-NC-ND 4.0

https://creativecommons.org/licenses/by-nc-nd/4.0/ 


\title{
Nadège Veldwachter, Littérature francophone et mondialisation
}

\author{
Jean-François Plamondon
}

\section{RÉFÉRENCE}

NADÈGE VELDWACHTER, Littérature francophone et mondialisation, Paris, Kathala, 2012, pp.

315.

Est-ce que ce livre est bien nommé? Peut-on véritablement parler de littérature francophone quand on ne se réfère qu'à la production littéraire de la francophonie africaine et antillaise? Ne devrions-nous pas embrasser la francophonie dans son ensemble quand le titre donne à croire qu'il sera question de toutes les littératures d'expression française à l'heure de la mondialisation? Nommée au singulier, la littérature francophone devient un concept englobant. Pourtant, les propos de Nadège Veldwachter démontrent qu'elle mise sur une scission du monde éditorial de la francosphère pour construire son argumentation: «Vu sous un angle sommairement schématisé, les auteurs francophones du Nord parmi lesquels se classent Canadiens, Belges, ou Suisses participent à une mécanique éditoriale armée d'outils de promotion et d'un réseau de distribution satisfaisant les besoins d'une industrie compétitive sur les principaux marchés internationaux. De l'autre, les francophones du Sud couvrant les régions subsaharienne, maghrébine et de l'est européen doivent évoluer dans un environnement adverse: analphabétisme, faiblesse du pouvoir d'achat, coûts de transport, librairies insuffisantes, bibliothèques mal équipées, etc» (p. 19). Je suis parfaitement d'accord avec cette analyse, et apprécie le fait que l'auteure spécifie que la situation décrite est «sommairement schématisée». Le fait que les Québécois aient un champ littéraire autonome et qu'ils fassent partie d'un pays riche ne change toutefois rien à la donne: leur littérature est peu reconnue dans le monde; leurs auteurs, si talentueux soient-ils, sont moins connus en France que tous les Confiant, Ben Jelloun, Condé, Schwarz-Bart, Chamoiseau, Glissant et combien d'autres issus de cette francophonie du Sud. Séparer ainsi la francophonie entre un monde favorisé qui ne 
connaitrait que des problèmes de riches et un monde sans ressource, me parait «fort en café», pour employer une expression chère à Gaston Miron. Le Québec, la Suisse, la Belgique vivent autant en périphérie de la France que les pays de la francophonie du Sud. Aucun de ces espaces nationaux ne bénéficie du prestige et de l'autorité symboliques et culturels de la France. Il y a quelque chose dans cette répartition des mondes qui va à l'encontre de ce que cherche à démontrer Veldwachter, dont une grande partie du travail consiste à illustrer les effets des interactions entre la francophonie du Sud et la France. Or le fait que l'Europe ignore l'une des œuvres de langue française les plus originales et représentatives du xxi siècle, celle de MarieClaire Blais, est à mes yeux aussi incompréhensible et dramatique que le peu de reconnaissance qu'obtient Frankétienne hors des frontières d'Haïti. Ces lacunes parlent évidemment beaucoup du lectorat hexagonal, en disent énormément sur les élites qui le forment et mettent en lumière des problèmes de compréhension ou de communication entre la France et le Québec et Haïti. Pourtant d'autres élites, comme le Suisse Auguste Viatte, rappellent aussi qu'un écrivain comme Emile Nelligan n'a pas «eu la reconnaissance proportionnelle à [sa] qualité auprès d'un public français et international du fait de la non-communication littéraire entre espaces francophones et français» (p. 75). L'essentiel de la production littéraire d'Emile Nelligan s'écrit entre 1896-1899 et les Québécois sont encore les seuls à connaître ses poèmes. Les problèmes des littératures de langue française ne s'étalent donc pas que sur l'axe nord-sud, l'axe est-ouest réserve aussi son lot de déceptions et de luttes. Dans cette ère globalisée, la francophonie doit se penser comme un tout, ses logiques d'action doivent être multilatérales mais univoques. C'est le poids symbolique des cultures de langue française qui en dépend, ce sont aussi les valeurs humanistes de ces cultures qui sont en jeu. Quand on n'est pas au sommet, il ne sert à rien de diviser et il me semble que nous aurions tous pu tirer profit d'une analyse globale des problèmes que rencontre toute la littérature francophone à l'ère de la mondialisation; puisque les maisons d'édition des pays riches francophones ne sortent pas indemnes de cette lutte de pouvoir internationale, et Veldwachter en est pleinement consciente.

Qu'à cela ne tienne, si le titre programmatique est trompeur, cela ne veut pas dire que le programme proposé soit mal traité. Au contraire, à la lecture de l'ouvrage de Madame Veldwachter, on réalise pleinement les effets de la mondialisation sur les littératures des petites communautés. Et ces effets ressentis ne sont pas nécessairement tous négatifs, comme dit l'auteure: «si le monopole de la production culturelle appartient à l'Occident, on assiste aujourd'hui à une sorte de renversement de phénomène où la production littéraire et artistique non occidentale est mondialement recherchée» (p. 89). Ce qui explique d'ailleurs en partie pourquoi on ne lit pas MarieClaire Blais, mais ce qui n'éclaire en rien le sort réservé à Frankétienne. Il faut dire aussi que la France a perdu un peu de son prestige, un prestige absolument nécessaire pour que fleurisse par ailleurs la francophonie dans le monde. Julia Kristeva explique cette perte d'influence par une forme de dépression collective qui suit une logique nihiliste (p. 108). Alors que Veldwachter cite un discours du candidat à la présidence Sarkozy pour montrer que le pouvoir politique tient la francophonie à cœur (p. 122), je me permets de nuancer en rappelant que plus que les mots, les actions de Nicolas Sarkozy peignent les intentions du personnage, et son discours à Québec en 2008 a démontré à quel point la francophonie n'était à ses yeux qu'un bilboquet bien inutile. En encourageant les Québécois à demeurer au sein de la fédération canadienne, Sarkozy reconnaissait la suprématie d'un pouvoir anglophone sur un pouvoir francophone et 
demandait à ce que l'ordre établi par les anglophones soit respecté par les francophones. C'était là une première dans l'histoire de la présidence de la cinquième république. Mais Kristeva parlait déjà d'un déclin de la France sous le régime chiraquien et c'est probablement en voulant profiter d'une brèche que des écrivains francophones (provenant du Sud, du Nord, de l'Est et de l'Ouest) ont signé le manifeste Pour une littérature monde en français dont Veldwachter analyse les effets avec perspicacité. Ce sont en fait les tensions du champ littéraire francophone face au nouveau contexte mondialisant qui ressortent dans ce chapitre où l'on prend conscience que les métiers de l'édition doivent s'adapter en fonction d'un nouvel espace, d'une échelle internationale qui est la nouvelle mesure de l'horizon d'attente: «nous sommes entrés dans l'ère de la marchandisation. Elle fait suite à celle de la commercialisation qui a couronné l'éditeur en tant que propriétaire, commanditaire ou concepteur du livre. La marchandisation, au contraire, veut que l'éditeur abdique en faveur du marché» (p. 160) et Eric Vigne de la NRF ajoute que cette stratégie d'action favorise «le formatage du produit pour le grand échange marchand, c'est l'équarrissage de l'imaginaire au profit des valeurs de l'échange marchand universel» (p. 160).

Littérature francophone et mondialisation est un très bon ouvrage et il propose encore plusieurs pistes qui portent à la réflexion. Son chapitre sur le marronnage littéraire est particulièrement riche et pertinent. Les littératures francophones, comme toutes les littératures font appel à la traduction pour leur diffusion. Les cas du français vers l'allemand ou du français vers l'anglais nous sont toujours plus connus que les cas de marronnage, qui nous font passer du créole au français. Les autotraductions se révèlent, à cet égard, fort problématiques dans ce cadre d'analyse et soulèvent des questions qui s'envolent largement au-dessus de celles de la francophonie pour atterrir dans les plaines de la traductologie en général. En faisant, comme elle le fait, intervenir Cassin, Lacan, Schleiermacher ou Meschonnic (pp. 283-284), Veltwachter montre bien que tout n'est pas traduisible et si l'on veut arriver à mieux communiquer l'hypotexte, l'hypertexte doit tenir compte de toutes les valeurs qui définissent une communauté. Dommage que le livre fut mal nommé! 